\title{
Exercise-Associated Hyponatremia, Hypernatremia, and Hydration Status in Multistage Ultramarathons
}

\author{
Brian J. Krabak, MD, MBA; Grant S. Lipman, MD; Brandee L. Waite, MD; Sean D. Rundell, DPT, PhD \\ From the Department of Rehabilitation, Orthopedics and Sports Medicine, University of Washington and Seattle Children's Sports Medicine, \\ Seattle, WA (Dr Krabak); the Department of Emergency Medicine, Stanford University School of Medicine, Stanford, CA (Dr Lipman); the \\ Department of Physical Medicine and Rehabilitation, University of California, Davis Sports Medicine, Sacramento, CA (Dr Waite); and the \\ Department of Rehabilitation Medicine, University of Washington, Seattle, WA (Dr Rundell).
}

Objective.-Dysnatremia and altered hydration status are potentially serious conditions that have not been well studied in multistage ultramarathons. The purpose of this study was to assess the incidence and prevalence of exercise-associated hyponatremia (EAH) $\left(\mathrm{Na}^{+}<135 \mathrm{mmol} \cdot \mathrm{L}^{-1}\right)$ and hypernatremia $\left(\mathrm{Na}^{+}>145 \mathrm{mmol} \cdot \mathrm{L}^{-1}\right)$ and hydration status during a multistage ultramarathon.

Methods.- This study involved a prospective observational cohort study of runners competing in a 250-km (155-mile) multistage ultramarathon (in the Jordan, Atacama, or Gobi Desert). Prerace body weight and poststage (stage [S] 1 [42 km], S3 [126 km], and S5 [250 km]) body weight and serum sodium concentration levels were obtained from 128 runners.

Results.-The prevalence of EAH per stage was $1.6 \%$ (S1), $4.8 \%$ (S3), and $10.1 \%$ (S5) with a cumulative incidence of $14.8 \%$. Per-stage prevalence of hypernatremia was $35.2 \%$ (S1), 20.2\% (S3), and $19.3 \%$ (S5) with a cumulative incidence of $52.3 \%$. Runners became more dehydrated (weight change $<-3 \%)$ throughout the race $(\mathrm{S} 1=22.1 \%$; $\mathrm{S} 3=51.2 \%$; $\mathrm{S} 5=53.5 \%)$. Body weight gain correlated with EAH $(r=-0.21, P=.02)$. Nonfinishers of S3 were significantly more likely to have EAH compared with finishers $(75 \%$ vs $5 \%, P=.001)$, but there was no difference in either EAH or hypernatremia between nonfinishers and finishers of S5.

Conclusions.- The incidence of EAH in multistage ultramarathons was similar to marathons and single-stage ultramarathons, but the cumulative incidence of hypernatremia was 3 times greater than that of EAH. EAH was associated with increased weight gain (overhydration) in early stage nonfinishers and postrace finishers.

Keywords: hyponatremia, hypernatremia, ultramarathon running, hydration

\section{Introduction}

Participation in ultramarathon races has steadily increased over the past decade. ${ }^{1}$ Races are typically $>42 \mathrm{~km}$ and occur in a single stage or in multiple stages on varied terrain. The diverse extreme locations of multistage ultramarathons (eg, deserts, mountains, river crossings) and the equipment (eg, food, backpacks) that athletes need to carry throughout the race expose the

Corresponding author: Brian J. Krabak, MD, MBA, FACSM, University of Washington Sports Medicine at Husky Stadium, 3800 Montlake Blvd NE, Box 354060, Seattle, WA 98195; e-mail: bkrabak@uw.edu.

Submitted for publication September 2016.

Accepted for publication May 2017. athletes to unique challenges and, potentially, serious illnesses. Serious causes of illness in this population include electrolyte disturbances, hydration imbalances, heat-related illness, and cardiovascular compromise. ${ }^{2-6}$

Dysnatremia has been an area of significant interest in this population of runners. The majority of research has focused on exercise-associated hyponatremia (EAH), which is a potentially serious condition. EAH, defined as a serum sodium concentration $\left(\left[\mathrm{Na}^{+}\right]\right)<135 \mathrm{mEq} / \mathrm{L}$, is recognized as relatively common in endurance running events. ${ }^{7}$ Symptoms can vary, ranging from nausea, vomiting, and headaches to altered mental status, seizures, and death. The incidence of EAH varies depending on the distance of the race, with reported rates of 3 to $28 \%$ for marathons, ${ }^{8,9} 23$ to $38 \%$ for 
triathlons, ${ }^{10}$ and 4 to $51 \%$ for single-stage ultramarathons. $^{1,11,12}$ However, there are no published incidence rates for multistage ultramarathons.

Despite being more common than EAH, markedly less is known about hypernatremia. ${ }^{7,11,13,14}$ Hypernatremia, defined as $\left[\mathrm{Na}^{+}\right]>145 \mathrm{mEq} / \mathrm{L}$, can present with symptoms similar to those of EAH, making it challenging to distinguish the 2 entities. ${ }^{1}$ The incidence rates of hypernatremia in single-stage ultramarathons are varied, reported in $<2 \%$ of all participants during the Western States 100 -Mile Endurance Run ${ }^{11}$ and 48 to $55 \%$ of collapsed runners in the Comrades Marathon. ${ }^{15}$ As with $\mathrm{EAH}$, there are no published incidence rates of hypernatremia for multistage ultramarathons.

Hydration imbalances in the long-distance runner have been another area of significant research. Several field studies have identified an association between weight gain and EAH in marathon runners. ${ }^{2,8,11,16,17}$ These studies would suggest that overconsumption of hypotonic fluids contributes to EAH, significant illness, and decreased performance in runners. In contrast, a study of single-stage ultramarathon runners noted that weight loss (ie, dehydration), especially in warm weather, had a weak but significant association with EAH. ${ }^{11}$ In addition, the authors reported a significant relationship between weight loss and performance, such that faster runners lost more weight. These findings are in contrast with controlled laboratory studies that have shown dehydration ( $>2 \%$ body weight) leads to a decrease in athletic performance, which is compounded by heat. ${ }^{18-20}$

Fortunately, research has shown that the majority of athletes who experience dysnatremia or hydration imbalances are often asymptomatic. In addition, symptomatic runners can usually recover quickly with appropriate interventions. ${ }^{7}$ Unfortunately, athlete characteristics have not been found helpful in risk stratification. ${ }^{3,7,11,21}$ When symptoms do occur, they may be confused with other causes, such as exercise-associated collapse, heat-related illness, or cardiovascular compromise. ${ }^{4-6}$ Delayed diagnosis and treatment could result in serious consequences, including seizures, pulmonary edema, or death. ${ }^{16,17,21}$ Therefore, understanding dysnatremia and hydration status in multistage ultramarathons is of the utmost importance.

Given the lack of information regarding dysnatremia and hydration in multistage ultramarathon runners, the present observational study sought to examine the serum blood sodium concentration levels and hydration status of athletes during such events to determine whether these values are similar to or different from other long-distance events. The aims of this study were to 1) prospectively analyze the incidence and prevalence of EAH and hypernatremia in multistage ultramarathons and 2) assess the relationship between poststage and postrace $\left[\mathrm{Na}^{+}\right]$ levels and the hydration status of finishers and nonfinishers.

\section{Methods}

\section{SETTING}

This study was conducted across several 4 Deserts multistage ultramarathons: the 2012 Atacama Crossing (Chile), the Sahara Race (Jordan), the Gobi March (China), and the 2013 Atacama Crossing (Chile). In each race, participants completed 4 consecutive stages of approximately $42 \mathrm{~km}$ (26 miles) per day followed by a fifth stage of approximately $80 \mathrm{~km}$ (50 miles) over 1 to 2 days. All races occurred over similar terrain, including deserts, river crossings, and slot canyons, with little trail or paved road. Participants started at a specific time each morning and completed the stage within an allotted time. Any participant who did not start a specific stage or dropped out of the race during the course of a stage for medical reasons was considered removed from competition (ie, nonfinisher). All participants were offered the same amount of water on any given day (approximately $1.5 \mathrm{~L}$ per $10-12 \mathrm{~km}$ ), had to carry at least $2000 \mathrm{kcal} / \mathrm{d}$ (verified during registration) of food, and did not receive any food beyond what they carried. Participants were required to carry all their personal items at all times throughout the entire race, including clothes, sleeping gear, emergency gear, and their supply of food.

Daily maximal temperature was collected using a Suunto Vector (Suunto, Finland) for each stage of a race. The climate conditions of the 4 races had relatively similar average maximal temperatures (Atacama 2012, $33.2 \pm 1.1^{\circ} \mathrm{C}$; Jordan, $32.1 \pm 1.4^{\circ} \mathrm{C}$; Gobi, $35.3 \pm 1.1^{\circ} \mathrm{C}$; Atacama 2013, 34.9 $\pm 1.6^{\circ} \mathrm{C}$ ). All races occurred in desert settings where the relative humidity is 10 to 20\%. The University of Washington and Stanford University School of Medicine Institutional Review Boards approved the study design.

\section{STUDY POPULATION}

All entrants competing in a 4 Deserts multistage ultramarathon (Atacama 2012 [ $n=148]$ ] Gobi 2012 [ $n=160]$; Sahara 2012 [ $n=134]$, and Atacama 2013 [n=148]) who understood English were invited to participate in the study. One month prior to the start of a race, all registered race participants received a recruitment flyer via email that described the study. At the time of race registration, study researchers reviewed the study with the interested participants and obtained informed consent. Participants were excluded from the study if they 
were aged $<18$ years or $>90$ years or were unable to read and understand English.

\section{STUDY PROTOCOL}

Prerace body weight and pack weight were measured prior to the start of each ultramarathon. Repeat body weight measurements were obtained immediately after completion of stage (S) 1 and S3 and postrace (S5) for each event or on removal from competition. Measurements of each runner were obtained at the stage finish line prior to consumption of any additional fluids. All measurements were made with a calibrated batterypowered digital scale (SC-505 HoMedics; Commerce Township, MI) placed on a solid, level surface. The digital scale was calibrated with a standard weight before and after measurements at each location to assure accuracy of measurements throughout the race.

At each race, an analysis of blood sodium concentration was obtained immediately after completion of S1, S3, and S5 or on removal from competition. Athletes did not consume food or water prior to testing. Each participant provided a venous blood sample while in a seated position. Blood was drawn into a heparinized capillary tube via finger prick. A point-of-care analyzer (i-STAT $6+$ cartridge; i-STAT system, Abbott Point of Care, Princeton, NJ) was used onsite to immediately measure blood sodium concentration to determine $\left[\mathrm{Na}^{+}\right]$levels. Handheld i-STAT blood analysis has been established as reliable compared with a standard laboratory electrolyte analyzer. ${ }^{22}$ The point-of-care device was calibrated for each stage of the race prior to measurements. Any participant who did not start or complete a stage was considered a nonfinisher.

\section{DEFINITIONS}

For comparison purposes, the following definitions were used based on the $\left[\mathrm{Na}^{+}\right]$and hydration status cutoff points used by Noakes ${ }^{16}$ and Hoffman ${ }^{11}$ : Hypernatremia was $>145 \mathrm{mmol} \cdot \mathrm{L}^{-1}$, normonatremia was 135 to $\leq 145$ $\mathrm{mmol} \cdot \mathrm{L}^{-1}$, biochemical hyponatremia was 129 to $<135$ $\mathrm{mmol} \cdot \mathrm{L}^{-1}$, and clinical significant hyponatremia was $<129 \mathrm{mmol} \cdot \mathrm{L}^{-1}$. Hydration status was based on body weight changes, with $\geq 0 \%$ body weight change as overhydration, $<0$ to $-3 \%$ body weight change as euhydration, and $<-3 \%$ body weight change as dehydration. Several articles have suggested that hydration status based on body weight changes is a positive indicator for overhydration. ${ }^{7,8,14,16,23}$

\section{STATISTICAL ANALYSIS}

A descriptive analysis was performed to evaluate the age, sex, height, baseline body weight, start pack weight, body mass index, and race time for each study participant. Serum sodium concentration, body weight, and the cumulative changes in both were described after S1, S3, and S5. We calculated the cumulative incidence of hyponatremia and hypernatremia and described the prevalence of hyponatremia, hypernatremia, and hydration status after S1, S3, and S5. Cumulative incidence was calculated as the proportion of racers who developed hyponatremia or hypernatremia at any point during the ultramarathon. We only included those who finished a stage when estimating the prevalence. Any participant who was unable to provide a sample or experienced a device malfunction was eliminated from this analysis. The relationship between postrace $\left[\mathrm{Na}^{+}\right]$and weight change was estimated by Pearson correlation coefficient. Logistic regression was used to test the association between dysnatremia and change in body weight, adjusted for age, sex, body mass index, pack weight, and race location. We tested the association between hyponatremia or hypernatremia and being a nonfinisher using Fisher exact tests. Because all races had approximately equal distances and similar maximal temperatures and logistical demands, the participants were combined into 1 cohort for analysis. Data are represented as mean \pm standard deviation. Statistical significance was considered at $P<.05$. All analyses were performed using Stata IC version 12.1 (College Station, TX).

\section{Results}

One hundred and fifty-two unique participants were recruited to participate in this study. Of these, 24 (Atacama 2012 [ $\mathrm{n}=12$ ]; Gobi 2012 [n=2]; Sahara 2012 $[\mathrm{n}=8]$; and Atacama 2013 [n=2]) did not complete the study protocol due to voluntary removal from the study $(n=4)$ or device error with blood sample testing $(n=20)$. The results of the remaining 128 participants were analyzed (Table 1), with a total of 380 individual blood samples collected over the study period.

The prevalence of EAH increased over the course of a race $(\mathrm{S} 1=1.6 \%, \mathrm{~S} 3=7.0 \%$, and $\mathrm{S} 5=11.3 \%)$, with a cumulative incidence of $14.8 \% \quad(n=19)$. The prevalence of hypernatremia decreased over the course of a race $(\mathrm{S} 1=35.2 \%, \mathrm{~S} 3=20.3 \%$, and $\mathrm{S} 5=21.8 \%)$, with a cumulative incidence of $52.3 \%(n=67)$. The body weight change over a race was $-2.8 \pm 2.7 \mathrm{~kg}(3.4 \pm 3.4 \%)$ for males and $-1.5 \pm 2.3 \mathrm{~kg}(2.4 \pm 3.7 \%)$ for females. After S1, $45.3 \%$ $(n=58)$ of study participants were overhydrated; $23.4 \%$ $(\mathrm{n}=30)$ after $\mathrm{S} 3$; and $16.1 \%(\mathrm{n}=20)$ postrace.

Table 2 shows the postrace measurements of study participants who participated in all stages of the race stratified into 4 different $\left[\mathrm{Na}^{+}\right]$states and 3 different hydration states. The majority of study participants 
Table 1. Characteristics of the participants

\begin{tabular}{|c|c|c|c|}
\hline & Males $(n=92)$ & Females $(n=36)$ & All participants $(n=128)$ \\
\hline Age $(\mathrm{y})^{a}$ & $39.9(9.3)$ & $38.9(7.9)$ & $39.6(8.9)$ \\
\hline Height $(\mathrm{m})^{a}$ & $1.81(0.13)$ & $1.68(0.73)$ & $1.78(0.13)$ \\
\hline Weight $(\mathrm{kg})^{a}$ & $79.5(9.9)$ & $61.9(9.5)$ & $74.5(12.6)$ \\
\hline $\operatorname{BMI}\left(\mathrm{kg} / \mathrm{m}^{2}\right)^{a}$ & $24.3(2.6)$ & $21.8(2.3)$ & $23.6(2.8)$ \\
\hline Pack weight $(\mathrm{kg})^{a}$ & $9.9(1.6)$ & $9.1(1.6)$ & $9.7(1.6)$ \\
\hline Race finish time $(\mathrm{h})^{a, b}$ & $43.5(8.7)$ & $48.0(12.1)$ & $44.7(9.8)$ \\
\hline Event & n $(\%)$ & n $(\%)$ & n $(\%)$ \\
\hline Atacama (2012) & $17(18.5)$ & $7(19.4)$ & $24(18.8)$ \\
\hline Jordan (2012) & $16(17.4)$ & $9(25.0)$ & $25(19.5)$ \\
\hline Gobi (2012) & $25(27.1)$ & $10(27.8)$ & $35(27.3)$ \\
\hline Atacama (2013) & $34(37.0)$ & $10(27.8)$ & $44(34.4)$ \\
\hline
\end{tabular}

${ }^{a}$ Values are mean (SD).

${ }^{b}$ Finishers only.

$(66.9 \%, \mathrm{n}=83)$ were normonatremic, of whom $54.2 \%$ $(\mathrm{n}=45)$ were dehydrated. In runners with dysnatremia, $21.8 \%(n=27)$ were hypernatremic and $11.3 \%(n=14)$ had EAH. The majority $(63.0 \%, \mathrm{n}=17)$ of runners with hypernatremia were dehydrated, whereas only $7.4 \%$ $(n=2)$ were overhydrated. In the group with EAH, $35.7 \%(n=5)$ were overhydrated, $50.0 \%(n=7)$ were dehydrated, and $14.3 \%(n=2)$ were euhydrated. Of the
$1.6 \%(\mathrm{n}=2)$ runners who experienced clinically significant hyponatremia, 1 was overhydrated and 1 was dehydrated.

The relationship between postrace $\left[\mathrm{Na}^{+}\right]$and change in body weight is shown in the Figure. There was a statistically significant correlation $(r=-0.21, P=.02)$ between postrace $\left[\mathrm{Na}^{+}\right]$and a change in body weight, with increased weight gain associated with a lower $\left[\mathrm{Na}^{+}\right]$.

Table 2. Classification of 124 athletes on the basis of 4 different serum $\left[\mathrm{Na}^{+}\right]$and 3 different states of hydration after racing a 250-km multistage ultramarathon

\begin{tabular}{|c|c|c|c|c|c|}
\hline Category & Hypernatremia & Normonatremia & $\begin{array}{c}\text { Biochemical } \\
\text { hyponatremia }\end{array}$ & $\begin{array}{c}\text { Clinically significant } \\
\text { hyponatremia }\end{array}$ & Total \\
\hline \multicolumn{6}{|l|}{ Overhydration } \\
\hline $\mathrm{n}$ & 2 & 13 & 4 & 1 & 20 \\
\hline$\% \mathrm{NA}^{+} \operatorname{group}^{a}($ column $\%)$ & 7.4 & 15.7 & 33.3 & 50.0 & \\
\hline$\% \mathrm{BW}$ group $^{b}$ (row $\left.\%\right)$ & 10.0 & 65.0 & 20.0 & 5.0 & \\
\hline$\%$ Total $($ cell \%) & 1.6 & 10.5 & 3.2 & 0.8 & 16.1 \\
\hline \multicolumn{6}{|l|}{ Euhydration } \\
\hline $\mathrm{n}$ & 8 & 25 & 2 & 0 & 35 \\
\hline$\% \mathrm{NA}^{+}$group (column $\%$ ) & 29.6 & 30.1 & 16.7 & 0.0 & \\
\hline$\% \mathrm{BW}$ group (row \%) & 22.9 & 71.4 & 5.7 & 0.0 & \\
\hline$\%$ Total $($ cell \%) & 6.5 & 20.2 & 1.6 & 0.0 & 28.2 \\
\hline \multicolumn{6}{|l|}{ Dehydration } \\
\hline $\mathrm{n}$ & 17 & 45 & 6 & 1 & 69 \\
\hline$\% \mathrm{NA}^{+}$group (column $\%$ ) & 63.0 & 54.2 & 50.0 & 50.0 & \\
\hline$\%$ BW group (row \%) & 24.6 & 65.2 & 8.7 & 1.5 & \\
\hline$\%$ Total $($ cell \%) & 13.7 & 36.3 & 4.8 & 0.8 & 55.7 \\
\hline \multicolumn{6}{|l|}{ Total } \\
\hline $\mathrm{n}$ & 27 & 83 & 12 & 2 & 124 \\
\hline$\%$ Total & 21.8 & 66.9 & 9.7 & 1.6 & 100.0 \\
\hline
\end{tabular}

${ }^{a} \%\left[\mathrm{Na}^{+}\right]$group refers to those with hypernatremia $\left(\left[\mathrm{Na}^{+}\right]>145 \mathrm{mmol} \cdot \mathrm{L}^{-1}\right)$, normonatremia $\left(\left[\mathrm{Na}^{+}\right] 135\right.$ to $\left.\leq 145 \mathrm{mmol} \cdot \mathrm{L}^{-1}\right)$, biochemical hyponatremia $\left(\left[\mathrm{Na}^{+}\right] 129\right.$ to $\left.<135 \mathrm{mmol} \cdot \mathrm{L}^{-1}\right)$, or clinically significant hyponatremia $\left(\left[\mathrm{Na}^{+}\right]<129 \mathrm{mmol} \cdot \mathrm{L}^{-1}\right)$ group.

${ }^{b} \% \mathrm{BW}$ group refers to those in a hydration status group based on body weight change of overhydration (weight change $\geq 0 \%$ ), euhydration (weight change $<0$ to $-3 \%$ ), or dehydration (weight change $<-3 \%$ ). 


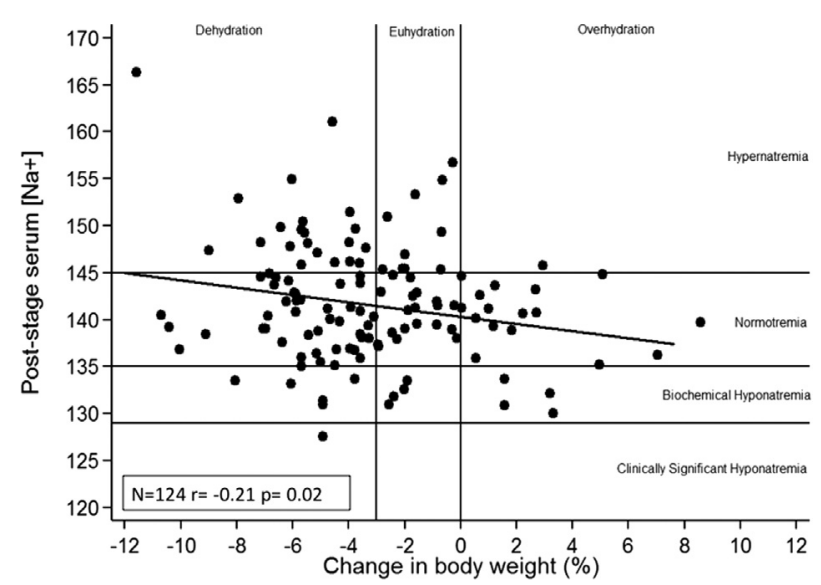

Figure. Relationship of postrace serum $\left[\mathrm{Na}^{+}\right]$with percentage change in body weight (postrace minus prerace) for 124 observations at 250-km multistage ultramarathons.

Each individual stage of the race revealed a nonsignificant relationship between $\left[\mathrm{Na}^{+}\right]$and body weight change from the prior stage: baseline to $\mathrm{S} 1$, $r=-0.15(P=.09)$; S1 to S3, $r=-0.16(P=.07)$; and S3 to $\mathrm{S} 5, r=-0.16(P=.08)$. The adjusted odds ratio of EAH postrace was 1.14 (95\% CI, 0.99-1.32) for every $1 \%$ increase in body weight, and the adjusted odds ratio of hypernatremia postrace was 1.10 (95\% CI, 0.93-1.30) for every $1 \%$ decrease in body weight.

A total of $10.1 \%(n=13)$ of study participants did not finish the race: 4 dropped out during S3 and 9 dropped out during S5. Of the S3 nonfinishers, $75 \%(\mathrm{n}=3)$ had EAH and $25 \%(n=1)$ were hypernatremic. All runners with EAH were overhydrated, and the runner with hypernatremia was euhydrated. Of the S5 nonfinishers, $44.4 \%(n=4)$ were hypernatremic, $33.3 \%(n=3)$ were normonatremic, and $22.2 \%(\mathrm{n}=2)$ had EAH. All S5 nonfinishers with hypernatremia were dehydrated, and those with EAH were equally likely to be overhydrated or dehydrated. There was a statistically significant relationship between EAH and S3 nonfinishers $(P=$ $.001)$, but not S5 nonfinishers $(P=.269)$. No significance was seen between hypernatremia and nonfinishers during either S3 $(P=1)$ or S5 $(P=.1)$. All nonfinishers experienced a variety of mild symptoms, including nausea, fatigue, and lightheadedness, at the time of dropping out of a race that resolved within 24 hours. None received IV hydration or experienced neurologic symptoms (eg, seizures or encephalopathy) or cardiopulmonary compromise (ie, respiratory distress).

\section{Discussion}

The current study is the first to establish incidence and prevalence rates of EAH and hypernatremia in multistage ultramarathons. The observed cumulative incidence of EAH was similar to that of marathons and single-stage ultramarathons. ${ }^{11,16}$ There was an increase in the prevalence of EAH as the race progressed, and a statistically significant correlation between postrace EAH and increased weight gain was found. Additionally, we found nonfinishers were more likely to experience EAH secondary to hypervolemia during the early, but not later, stages of a multistage ultramarathon. Despite the inherent challenges of using body weight as a marker of hydration status, multiple studies and a recent consensus statement on hyponatremia have supported the concept that increased body weight compared to baseline is a positive predictor of fluid overload in the running population. ${ }^{7,8,16}$

The relationship between EAH and hypervolemia observed during the earlier stages (marathon distances) of a multistage ultramarathon appears to be consistent with previous research that supports the concept of a dilutional mechanism for EAH. In this model, hyponatremia is secondary to weight gain primarily caused by overhydration from excessive fluid consumption. ${ }^{3,7,16,17}$ In addition, inadequate suppression of antidiuretic hormone secretion (arginine vasopressin) affected by nonosmotic stimuli, including excessive exercise, nausea, vomiting, pain, and plasma volume contraction, could contribute to a reduction in the excretion of free water from the kidneys. ${ }^{2,3,7,16,17}$ Similarly, studies of marathon runners and triathletes have shown a significant inverse linear association between $\left[\mathrm{Na}^{+}\right]$and percent change in body weight such that weight gain correlates with worsened EAH. ${ }^{8,16}$

Several factors unique to the multistage ultramarathon may contribute to the potential development of EAH in the setting of overconsumption of fluids during a race. In this setting, athletes must balance the daily physical cumulative stress of running with their daily nutritional recovery needs over the course of a race. ${ }^{1,2,24-26}$ Athletes with overzealous concern regarding dehydration during or after completion of a stage may consume excessive fluids in the hope of maintaining hydration over the course of the race. Given the extreme temperatures and arid conditions of a desert, runners may increase their fluid consumption due to increased insensible losses and greater hydration needs and to compensate for the dehydration that has been linked with increased physiologic strain and increased perceived exertion. ${ }^{20,27}$ Finally, excessive drinking may be reinforced by the belief that electrolyte supplementation may prevent EAH, despite the fact that studies do not support this belief. ${ }^{28-30}$ Although our findings set the foundation for understanding EAH in the multistage ultramarathon, future studies are necessary to further evaluate the effect of each of these factors. 
The prevalence of EAH was highest after the last and longest stage (ultramarathon distance) of the race, when athletes were most likely to be dehydrated (58\%). This finding is similar to those of a single-stage ultramarathon study in which EAH was slightly more predominant with dehydration than with overhydration. ${ }^{11}$ The authors proposed a depletional model for hyponatremia, where hot ambient temperatures led to dehydration-induced sodium depletion, secondary to inadequate suppression of antidiuretic hormone secretion (arginine vasopressin), impaired mobilization of osmotically inactive sodium stores, and/or inappropriate inactivation of osmotically active sodium. Potential contributing factors unique to both single-stage and multistage ultramarathons include excessive running distances, limited access to fluids, and propensity to experience gastrointestinal symptoms (nausea and vomiting) compared with shorter-distance races. ${ }^{1,3,7,26,31}$

We found that participants were 3 times more likely to experience hypernatremia than to experience EAH. Surprisingly, hypernatremia was much more prevalent than previously reported for both marathons and singlestage ultramarathons. ${ }^{11,16}$ Similar to prior studies of marathons, but not single-stage ultramarathons, the majority of the hypernatremic runners in the present study were dehydrated. ${ }^{11,29} \mathrm{~A}$ high incidence of hypernatremia $(45-58 \%)$ has been documented in collapsed runners of single-stage ultramarathons, who were significantly less likely to tolerate oral hydration and had a longer length of stay in medical tents than those with EAH. ${ }^{13,15,32}$ Hypernatremia has been often underemphasized in the ultramarathon literature, and the potential consequences of severe hypernatremia remain relatively underappreciated and unknown in this at-risk population.

The principle cause of the hypernatremia seen in this cohort of athletes remains unclear. Hypernatremia occurs when there is a deficit of total body water relative to total body sodium content. ${ }^{33}$ It is thought to be secondary to either an impaired thirst mechanism or limited access to water. It is theorized that exercise-associated hypernatremia may be compounded by a suppression of the thirst mechanism from prolonged exercise, exerciseinduced gastroparesis, or nausea in endurance athletes. ${ }^{32,34}$ In addition, hypernatremia may be influenced by food and fluid intake, although further studies are needed. ${ }^{28-30}$ In the multistage ultramarathon, it is possible that the cumulative effects of running long distances on successive days and the inability to fully recover may contribute to dehydration-induced hypovolemia, resulting in hypernatremia. This was evident in our cohort, which experienced an increase in dehydration as the race progressed and a statistically significant correlation between weight loss and increase in serum sodium levels. Although we were unable to fully evaluate all the variables in this relationship and the cause of hypernatremia in our studied population, further evaluation of contributing factors is an area for future research.

Overhydration has been established as an indicator for detection of fluid overload in the running athlete. ${ }^{7,23}$ In the current study, there was a relatively lower incidence of overhydration compared with the single-stage ultramarathon (16 vs 36\%). ${ }^{11}$ The lower rate may relate to several factors unique to the multistage ultramarathon race. As previously noted, multistage ultramarathon athletes have the ability to replenish food and fluids on a daily basis, whereas single-stage athletes may focus more on maintaining fluid consumption during the race. Multistage athletes may have minimized the amount of fluid intake during the race, knowing they could rehydrate overnight. In addition, the incidence could have been affected by our prerace briefing, which highlights the risks of overconsumption of fluids.

The interpretation of dehydration should be done cautiously. Unlike weight gain, loss of body weight is an imperfect predictor of total body volume status. ${ }^{3,7,23}$ Field studies have suggested that greater weight loss (as a proxy for dehydration) has been seen in the most competitive finishers of endurance events, ${ }^{11,35,36}$ which is in contrast with controlled studies that have shown dehydration consistently decreases athletic performance. ${ }^{18-20}$ In our study, the incidence of dehydration was $36 \%$ higher compared with single-stage ultramarathons. ${ }^{11}$

Overall, the findings of the current study provide greater insight into the potential medical care of the multistage ultramarathon runner. Our results support the recommendation that caution is warranted prior to administration of IV fluids in the collapsed runner who cannot tolerate oral fluids because the likelihood of EAH, hypernatremia, and other serious causes of exercise-associated collapse (ie, dehydration, heatrelated illness, and cardiopulmonary compromise) may be clinically difficult to distinguish., ${ }^{3,6,7,26,37}$ When possible, point-of-care testing should be used to establish a serum sodium level, with treatment as appropriate. However, access to functional point-of-care blood testing to guide resuscitative fluid choices may be challenging in these races because the extreme temperatures, remote locations, and expense of this equipment may make it difficult to have functional equipment on hand. Therefore, IV fluids of any type (isotonic or hypertonic) should be used judiciously when testing is not available, given the greater incidence of hypernatremia compared with hyponatremia and the varying hydration states in the multistage ultramarathon runner. Future research is needed to assess the impact of current recommendations 
of hypertonic IV fluids on hypovolemic hypernatremia and hypovolemia with unknown blood sodium concentration levels in this population.

\section{LIMITATIONS}

This study has a few limitations worth noting. Blood sodium concentration levels and body weight were not measured prior to the start of a stage. A previous pilot study by the authors that attempted to collect measurements prior to the start of a race or stage resulted in significant participant drop out $(>75 \%)$. The main reasons noted were lack of time to collect and process the blood samples and impact on racers' ability to prepare for the start. Similar logistical issues have been cited as limitations in previous ultramarathon studies that attempted to collect prerace sodium concentration levels $^{11}$ and renal function levels, ${ }^{38}$ with the latter experiencing a $50 \%$ attrition rate over the course of the study.

Daily caloric and water intake, sodium sweat rates, antidiuretic hormone and plasma volumes levels, and sodium ingestion throughout the race were not determined. A lack of understanding regarding these entities may have confounded the results and conclusions of the study. Measuring these factors could provide a more accurate reflection of sodium homeostasis and hydration status in this population. However, we believe our findings provide a foundation for future studies because our methodology is the same as previous wellestablished studies of marathon and single-stage ultramarathon runners. ${ }^{11,16}$ Finally, a small number of participants were analyzed for each ultramarathon race, requiring grouping of the athletes into 1 cohort for analysis. Because each race was similar in design, length, and logistical demands, we feel this is appropriate because prior studies have combined athletes from different races for greater numbers and analysis. ${ }^{11,16,38}$

\section{Conclusion}

The incidence of EAH in multistage ultramarathons was similar to that in previous studies of marathon and single-stage ultramarathons, whereas hypernatremia was 3 times more common in multistage events. EAH was significantly associated with increased weight gain in early stage nonfinishers and postrace finishers. However, this relationship was not significant for late-stage nonfinishers, who were 2 times more likely to be hypernatremic than hyponatremic. Educating athletes and medical staff further regarding the likelihood of EAH vs hypernatremia during a multistage ultramarathon should assist in the evolution and optimization of fluid replacement strategies. Additionally, future studies are needed to better understand the mechanism of and identify the factors that contribute to dysnatremia in the multistage ultramarathon and optimize treatment.

Acknowledgments: The authors thank Drs David Young, Colin Little, Natalie Badowski, and Richard Yoon for assistance with the study. They also thank all the athletes, without whom this study would not have been possible, for their participation. The study was supported by the RacingThePlanet Research Grant, and Abbott provided the material support of the i-STAT devices.

Author Contributions: Study concept and design (BJK, GSL, BLW); acquisition of the data (BJK, GSL); analysis of the data (SDR, BJK, GSL); drafting of manuscript (BJK, GSL, BLW, SDR); critical revision of the manuscript (BJK, GSL, BLW, SDR); and approval of final manuscript (BJK).

Financial/Material Support: None.

Disclosures: None.

\section{References}

1. Krabak BJ, Waite BW, Lipman GS. Evaluation and treatment of injury and illness in the ultramarathon athlete. Phys Med Rehabil Clin N Am. 2014;25:845-863.

2. Krabak BJ, Waite B, Lipman G. Injury and illnesses prevention for ultramarathoners. Curr Sports Med Rep. 2013;12:183-189.

3. Hoffman MD, Pasternak A, Rogers IR, et al. Medical services at ultra-endurance foot races in remote environments: medical issues and consensus guidelines. Sports Med. 2014;44:1055-1069.

4. Lipman GS, Eifling KP, Ellis MA, et al. Wilderness Medical Society practice guidelines for the prevention and treatment of heat-related illness: 2014 update. Wilderness Environ Med. 2014;25(suppl 4):S55-S65.

5. Spano SJ, Reagle Z, Evans T. Symptomatic hypotonic hyponatremia presenting at high altitude. Wilderness Environ Med. 2014;25:69-74.

6. Bennett BL, Hew-Butler T, Hoffman MD, Rogers IR, Rosner MH. Wilderness Medical Society practice guidelines for treatment of exercise-associated hyponatremia: 2014 update. Wilderness Environ Med. 2014;25(suppl 4):S30-S42.

7. Hew-Butler T, Rosner MH, Fowkes-Godek, et al. Statement of the Third International Exercise-Associated Hyponatremia Consensus Development Conference, Carlsbad, California, 2015. Clin J Sport Med. 2015;25:303-320.

8. Almond CS, Shin AY, Fortescue EB, et al. Hyponatremia among runners in the Boston Marathon. $N$ Engl J Med. 2005;352:1550-1556.

9. Roberts WO. A 12-yr profile of medical injury and illness for the Twin Cities Marathon. Med Sci Sports Exerc. 2000; 32:1549-1555.

10. Speedy BD, Noakes TD, Rogers IR, et al. Hyponatremia in ultradistance athletes. Med Sci Sports Exerc. 1999;31:809-815.

11. Hoffman MD, Hew-Butler T, Stuempfle KJ. Exerciseassociated hyponatremia and hydration status in 161-km ultramarathoners. Med Sci Sports Exerc. 2013;45: 784-791. 
12. Cairns RS, Hew-Butler T. Incidence of exercise-associated hyponatremia and its association with nonosmotic stimuli of arginine vasopressin in the GNW100s ultra-endurance marathon. Clin J Sport Med. 2015;25:347-354.

13. Siegel AJ, d'Hemecourt P, Adner MM, Shirey T, Brown JL, Lewandroski KB. Exertional dysnatremia in collapsed marathon runners: a critical role for point-of-care testing to guide appropriate therapy. Am J Clin Pathol. 2009;132:336-340.

14. Hoffman MD, Stuempfle KJ, Valentino T. Sodium intake during an ultramarathon does not prevent muscle cramping, dehydration, hyponatremia, or nausea. Sports Med Open. 2015;1:39.

15. Hew-Butler T, Sharwood K, Boulter J, et al. Dysnatremia predicts a delayed recovery in collapsed ultramarathon runners. Clin J Sport Med. 2007;17:289-296.

16. Noakes TD, Sharwood K, Speedy D, et al. Three independent biological mechanisms cause exercise-associated hyponatremia: evidence from 2,135 weighed competitive athletic performances. Proc Natl Acad Sci USA. 2005;102: 18550-18555.

17. Krabak BJ, Parker KM, DiGirolamo A. Exercise associated collapse: is hyponatremia in our head? PM R. 2016;8 (suppl 3):S62-S68.

18. Armstrong LE, Costill DL, Fink WJ. Influence of diureticinduced dehydration on competitive running performance. Med Sci Sports Exerc. 1985;17:456-461.

19. Ebert TR, Martin DT, Bullock N, et al. Influence of hydration status on thermoregulation and cycling hill climbing. Med Sci Sports Exerc. 2007;39:323-329.

20. Casa DJ, Armstrong LE, Hillman SK, et al. National Athletic Trainers' Association position statement: fluid replacement for athletes. J Athl Train. 2000;35:212-224.

21. Hoffman MD, Fogard K, Winger J, Hew-Butler T, Stuempfle KJ. Characteristics of 161-km ultramarathon finishers developing exercise-associated hyponatremia. Res Sports Med. 2013;21:164-175.

22. Erickson KA, Wilding P. Evaluation of a novel point-ofcare system, the i-STAT portable clinical analyzer. Clin Chem. 1993;39:283-287.

23. Stachenfeld NA. The interrelationship of research in the laboratory and the field to assess hydration status and determine mechanisms involved in water regulation during physical activity. Sports Med. 2014;44(suppl 1):S97-S104.

24. Bürge J, Knechtle B, Knechtle P, Gnädinger M, Rüst CA, Rosemann T. Maintained serum sodium in male ultramarathoners-the role of fluid intake, vasopressin, and aldosterone in fluid and electrolyte regulation. Horm Metab Res. 2011;43:646-652.

25. Knechtle B, Senn O, Imoberdorf R, et al. Maintained total body water content and serum sodium concentrations despite body mass loss in female ultra-runners drinking ad libitum during a $100 \mathrm{~km}$ race. Asia Pac J Clin Nutr. 2010;19:83-90.

26. Krabak BJ, Waite B, Schiff MA. Study of injury and illness rates in multiday ultramarathon runners. Med Sci Sports Exerc. 2011;43:2314-2320.

27. American Dietetic Association; Dieticians of Canada; American College of Sports Medicine. Rodriguez NR, Di Marco NM, Langley S. American College of Sports Medicine position stand. Nutrition and athletic performance. Med Sci Sports Exerc. 2009;41:709-731.

28. Freda B, Nurko S. Hyponatremia and hypernatremia. In: Nurko S, ed. Cleveland Clinic: Current Clinical Medicine. 2nd ed. Cleveland, OH: Saunders, Elsevier; 2010:818-823.

29. Hoffman MD, Stuempfle KJ. Sodium supplementation and exercise-associated hyponatremia during prolonged exercise. Med Sci Sports Exerc. 2015;47:1781-1787.

30. Hew-Butler TD, Sharwood K, Collins M, Speedy D, Noakes T. Sodium supplementation is not required to maintain serum sodium concentrations during an Ironman triathlon. Br J Sports Med. 2006;40:255-259.

31. Stuempfle KJ, Hoffman MD. Gastrointestinal distress is common during a 161-km ultramarathon. J Sports Sci. 2015;33:1814-1821.

32. Hew-Butler T, Boulter J, Godlonton J, Tucker R, Noakes T. Hypernatremia and intravenous fluid resuscitation in collapsed ultramarathon runners. Clin J Sport Med. 2008;18: 273-278.

33. Overgaard-Steensen C, Ring T. Clinical review: practical approach to hyponatraemia and hypernatraemia in critically ill patients. Crit Care. 2013;17:206.

34. Hoffman MD, Wegelin JA. The Western States 100-Mile Endurance Run: participation and performance trends. Med Sci Sports Exerc. 2009;41:2191-2198.

35. Zouhal H, Groussard C, Minter G, et al. Inverse relationship between percentage body weight change and finishing time in 643 forty-two-kilometre marathon runners. $\mathrm{Br} \mathrm{J}$ Sports Med. 2011;45:1101-1105.

36. Sharwood KA, Collins M, Goedecke JH, Wilson G, Noakes TD. Weight changes, medical complications, and performance during an Ironman triathlon. Br J Sports Med. 2004;38:718-724.

37. McGarvey J, Thompson J, Hanna C, Noakes TD, Stewart J, Speedy D. Sensitivity and specificity of clinical signs for assessment of dehydration in endurance athletes. $\mathrm{Br} \mathrm{J}$ Sports Med. 2010;44:716-719.

38. Lipman GS, Krabak BJ, Rundell SD, Shea KM, Badowski $\mathrm{N}$, Little C. Incidence and prevalence of acute kidney injury during multistage ultramarathons. Clin J Sport Med. 2016;26:314-319. 